\title{
Hybrid computed tomography and magnetic resonance imaging 3D printed models for neurosurgery planning
}

\author{
Teodoro Martín-Noguerol ${ }^{1}$, Félix Paulano-Godino ${ }^{1}$, Roy F. Riascos ${ }^{2}$, Juan Calabia-del-Campo ${ }^{3}$, \\ Javier Márquez-Rivas ${ }^{4}$, Antonio Luna ${ }^{1}$
}

${ }^{1}$ MRI Unit, Radiology Department, HT Médica, Jaén, Spain; ${ }^{2}$ Department of Neuroradiology, The University of Texas Health Science Center at Houston, McGovern Medical School, Texas, USA; ${ }^{3}$ Radiology Department, Hospital Clínico Universitario de Valladolid, Valladolid, Spain; ${ }^{4}$ Neurosurgery Service, Hospital Universitario Virgen del Rocío, Sevilla, Spain

Contributions: (I) Conception and design: T Martín-Noguerol, F Paulano-Godino, A Luna; (II) Administrative support: None; (III) Provision of study materials or patients: T Martín-Noguerol, F Paulano-Godino, A Luna; (IV) Collection and assembly of data: T Martín-Noguerol, F PaulanoGodino; (V) Data analysis and interpretation: None; (VI) Manuscript writing: All authors; (VII) Final approval of manuscript: All authors.

Correspondence to: Teodoro Martín-Noguerol, MD. MRI Unit, Radiology Department, HT Médica, Carmelo Torres 2, 23007 Jaén, Spain. Email: t.martin.f@htime.org.

\begin{abstract}
In the last decade, the clinical applications of three-dimensional (3D) printed models, in the neurosurgery field among others, have expanded widely based on several technical improvements in $3 \mathrm{D}$ printers, an increased variety of materials, but especially in postprocessing software. More commonly, physical models are obtained from a unique imaging technique with potential utilization in presurgical planning, generation/creation of patient-specific surgical material and personalized prosthesis or implants. Using specific software solutions, it is possible to obtain a more accurate segmentation of different anatomical and pathological structures and a more precise registration between different medical image sources allowing generating hybrid computed tomography (CT) and magnetic resonance imaging (MRI) 3D printed models. The need of neurosurgeons for a better understanding of the complex anatomy of central nervous system (CNS) and spine is pushing the use of these hybrid models, which are able to combine morphological information from CT and MRI, and also able to add physiological data from advanced MRI sequences, such as diffusion-weighted imaging (DWI), diffusion tensor imaging (DTI), perfusion weighted imaging (PWI) and functional MRI (fMRI). The inclusion of physiopathological data from advanced MRI sequences enables neurosurgeons to identify those areas with increased biological aggressiveness within a certain lesion prior to surgery or biopsy procedures. Preliminary data support the use of this more accurate presurgical perspective, to select the better surgical approach, reduce the global length of surgery and minimize the rate of intraoperative complications, morbidities or patient recovery times after surgery. The use of $3 \mathrm{D}$ printed models in neurosurgery has also demonstrated to be a valid tool for surgeons training and to improve communication between specialists and patients. Further studies are needed to test the feasibility of this novel approach in common clinical practice and determine the degree of improvement the neurosurgeons receive and the potential impact on patient outcome.
\end{abstract}

Keywords: Three-dimensional printing (3D printing); magnetic resonance imaging (MRI); computed tomography (CT); hybrid imaging; neurosurgery; brain; spine

Submitted Sep 21, 2019. Accepted for publication Oct 29, 2019.

doi: 10.21037/atm.2019.10.109

View this article at: http://dx.doi.org/10.21037/atm.2019.10.109 


\section{Introduction}

The first applications of three-dimensional (3D) printing in medicine appeared in the nineties. In the last decade the use of printed physical models is experimenting with exponential growth, related to several internal and external factors affecting all the steps of the $3 \mathrm{D}$ printing workflow $(1,2)$. The overall improvement in the spatial resolution of radiological images enables the generation of highquality $3 \mathrm{D}$ reconstructions. New multidetector computed tomography (CT) or magnetic resonance imaging (MRI) equipment can provide morphological and functional information within the same protocol. Recent advances in imaging segmentation and post-processing software are allowing the generation of $3 \mathrm{D}$ virtual and printable models in a more accurate manner (3). Moreover, new engineering developments in the field of $3 \mathrm{D}$ printers and materials are enabling us to transform these virtual 3D models into real physical designs. Besides, 3D printing software is beginning to be introduced in the clinical imaging work-flow of radiologists and other specialists working with imaging making accessible for them to generate physical models. Finally, another reason for this new wave in $3 \mathrm{D}$ printing is a growing need, arising from physicians and especially from surgeons, of going one step further in their patients' care (4). In this manner, the applications of $3 \mathrm{D}$ printing have expanded from surgical planning and surgical simulation to the generation of personalized surgical material $(5,6)$.

The use of $3 \mathrm{D}$ printed models is allowing a better understanding of patient's anatomy and pathology. Moreover, 3D models are changing the planning of surgical procedures, with several direct benefits, such as increased safety of surgical procedures with reduction of surgical time and complications, which is also enhanced with the use of personalized surgical material $(6,7)$. Furthermore, physical models improve communication between surgeons and patients, providing them a better understanding of their clinical case and need for surgery. The way how surgeons acquire or train their skills is also improved with the introduction of this technology (5).

In the field of neurosurgery, all of these items become even more relevant due to the intrinsic complexity of brain and spine anatomy, the variety of pathological conditions which may need to be surgically treated and the not negligible risk linked to these surgical procedures (8). For all these reasons, the development of $3 \mathrm{D}$ printing models in neurosurgery is becoming a reality in common practice. However, there are some limitations in the generation process of additive manufactured models for neurosurgical applications. Most of the time 3D printing models for common clinical applications are created using CT as an imaging source, as some anatomical structures are easily segmented, such as bone (2). However, MRI provides a better tissue contrast to discriminate with high accuracy between lesions and soft tissues in the brain and spine (9). In this manner, merging data from both imaging modalities permits to obtain the best information from each one, permitting to generate more realistic and accurate hybrid physical models. Moreover, advanced MRI using sequences like functional MRI (fMRI) based on blood oxygenation level dependent (BOLD) imaging, diffusion-weighted imaging (DWI), diffusion tensor imaging (DTI) or perfusion weighted imaging (PWI), offer additional information about tissue physiopathology (10). These advanced imaging techniques can provide histopathological information beyond conventional anatomic landmarks from morphological acquisitions. In this manner, the cellularity or neoangiogenesis of central nervous system (CNS) or spine lesions can be estimated using DWI or PWI, respectively. The combined use of these functional data with structural information potentially enhances 3D models, which can include all the information needed for a proper planification of the different surgical options. This way has been scarcely explored in clinical practice as the needs of particular adjustments in acquisition protocols and specific fusion software for achieving a proper registration of data from different sources.

In this review, a brief analysis of the current state of $3 \mathrm{D}$ modeling and printing in neurosurgery is performed. Technical tips to optimally perform hybrid physical models using both structural and functional information form CT and MRI and a resume of their potential clinical applications are also detailed.

\section{Current state of 3D printing in neurosurgery}

Recent technological advances in the segmentation and printing process have impulse the introduction of additive manufactured models in neurosurgery. The development of software based on artificial intelligence, particularly machine learning and deep learning, is improving the segmentation process of medical images (11). This segmentation step is crucial for accurate discrimination between different anatomical regions, tissues and most importantly, to distinguish between normal and pathological structures (2). Another important pillar that has supported the hype of $3 \mathrm{D}$ printing is the development of new and 
Table 1 Main features of the current 3D printing technologies

\begin{tabular}{|c|c|c|c|c|c|}
\hline Features & Material extrusion & Vat photopolymerization & Material jetting & Binder jetting & Laser sintering \\
\hline Materials & Thermoplastics & Resins & Resins & $\begin{array}{l}\text { Ceramic, polyamides, } \\
\text { metals }\end{array}$ & Polyamides, metals \\
\hline $\begin{array}{l}\text { Minimum Z } \\
\text { resolution } \\
(\mathrm{mm})\end{array}$ & 0.15 & 0.025 & 0.014 & 0.08 & 0.02 \\
\hline
\end{tabular}

The minimum resolutions are approximate, since they depend on each printer model. Asterisks provide a visual approach about the cost of each technology group ranging from cheapest $\left({ }^{*}\right)$ to most expensive $\left(^{\star \star \star *}\right)$.

more advanced $3 \mathrm{D}$ printers and the wide diversity of printing materials (12). These new materials are allowing to print characteristics of human anatomy or pathology, not only using different colors but also playing with diverse texture, consistency and stiffness (13). Even now, new materials are biocompatible (14), opening a door for new applications, as the combination of stem cells with custom scaffolds for personalized regenerative medicine $(15,16)$. Table 1 summarizes the main features of the current $3 \mathrm{D}$ printing technologies.

The most common application of $3 \mathrm{D}$ printing is the use of physical models for surgical planning and training. Currently, the different types of material and 3D printers enable to print neurosurgical models, with the opportunity to assign different physical characteristics to each of the different CNS or spine tissues (bone, brain tissue, spinal cord, lesions...) or even represent the functional features of lesions (hypercellularity, neoangiogenesis). The high anatomical complexity of CNS and spine, together with the wide range of possible pathologies that neurosurgeons face every day, claims the development of new imaging-based realistic approaches. Moreover, the existence of multiple anatomical interindividual variations and the broad range of possible manifestations of the same disease may condition substantial changes in the surgical procedure and what is most important, in the patient outcome. In this manner, the use of patient-customized 3D printed models for surgical planning facilitates a truly individual and personalized surgical treatment in the precision medicine era.

Several advantages have been described related to the use of 3D printing models. 3D printing has demonstrated its value as a complementary tool for evaluation of radiological images allowing neurosurgeons to perform a global idea of the internal and external references of a certain pathology beyond conventional 3D multiplanar reconstructions $(1,17)$. Moreover, the use of $3 \mathrm{D}$ printing models is allowing better communication and exchange of information between neuroradiologists, neurosurgeons and neurologists (18). The use of $3 \mathrm{D}$ printed models for training of surgeons, residents, and medical students is increasing its popularity in the last years $(19,20)$. Therefore, their use in the academic arena for educational purposes is being more and more accepted and included in training programs (5) (Figure 1).

Previous studies, and our own clinical experience, support the idea that the more complex the evaluated anatomical region or lesion is, the higher the benefit from the use of a $3 \mathrm{D}$ printed model for surgical planning $(18,21)$. In these cases, the profit margin for the patient increases almost in 

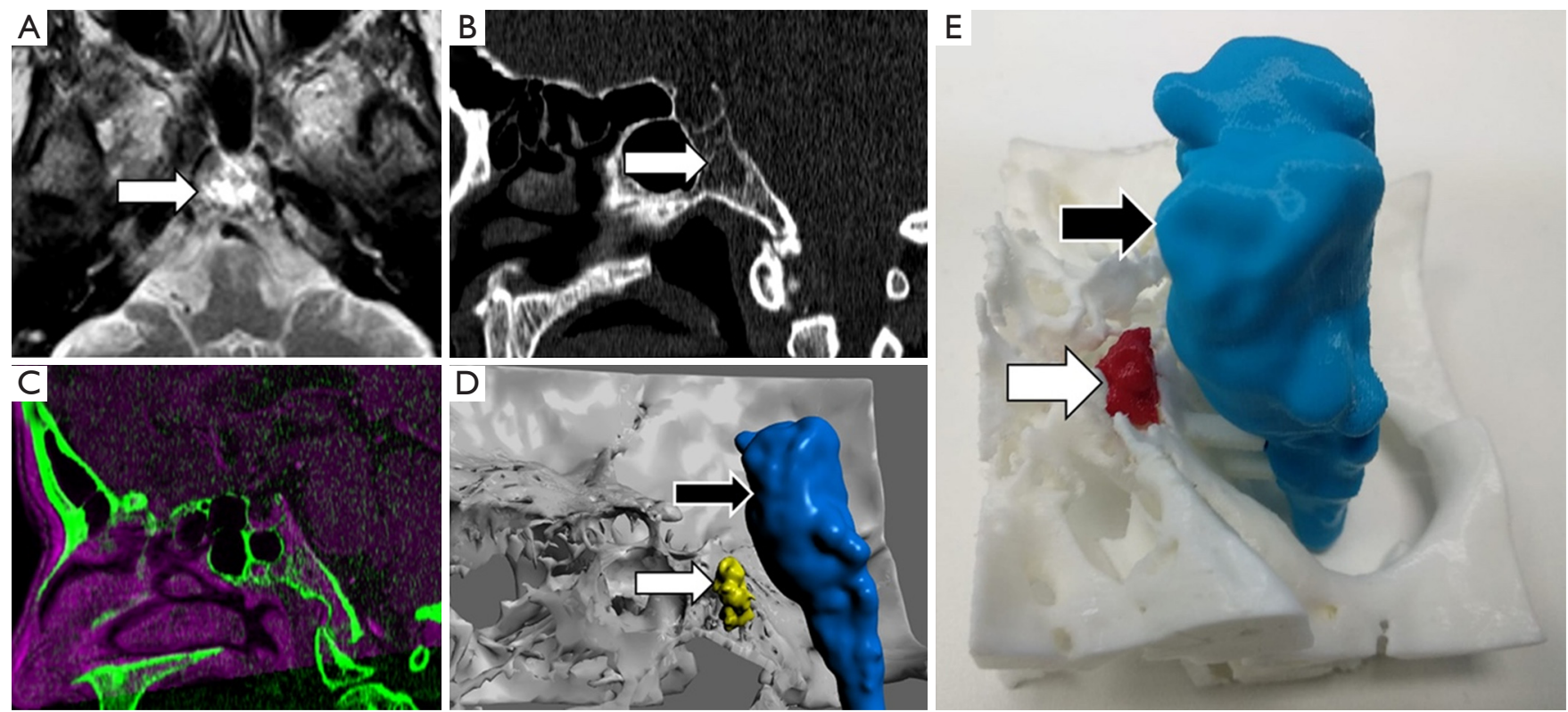

Figure 1 Clival chordoma evaluation. A 45-year-old female that undergone MRI for migraine. (A) Axial T2-TSE sequence shows hyperintense lesion within clivus within punctate hypointensities (arrow). (B) Sagittal reconstruction of CT confirms the presence of a lytic lesion within clivus s with probably chondroid matrix inside (arrow). (C) Registration of both MRI and MCDT information allows delimitating brain tissue (magenta) using information from MRI and skull base structures (green) from CT. (D) The 3D virtual model and (E) the 3D printed model enable neurosurgeon to properly identify the location of chordoma (white arrow) concerning the brainstem (black arrow) and the rest of the skull base. This approach allows reduction of the risk of neurological complications before surgery or biopsy.

an exponential manner when 3D printed models are used. Thus, interventions of skull-based, craniovertebral junction, spine or spinal cord lesions will benefit more from the use of physical models rather than other types of conventional surgical procedures (22) (Figure 2). In the case of complex lesions, 3D printing has demonstrated that can facilitate the surgical approach planning, providing neurosurgeons physical interaction with real patient anatomy determining before surgery which is the best surgical option (23). This circumstance will reduce the overall surgical procedure duration.

Some certified centers are going one step further by printing personalized surgical guides which reduction of procedure times (24). These guides are designed using specific software and fit perfectly into patient anatomy providing a safer and more accurate manner to guide the insertion of external devices, from neurostimulators to spine screws (25-28). The use of these types of personalized guides also contributes to the reduction of surgical times compared with conventional approaches $(29,30)$.

All of the items referred above benefit not only neurosurgeons but also patients. The reduction of the operative time is linked to shorter anesthesia times and less chance of surgical related complications (31). Moreover, shorter post-surgical recovery times have been described in patients when $3 \mathrm{D}$ printed models are used before surgery (32). The use of physical models leads to both, a patients' better understanding of their pathology, and a reduction in their possible doubts about the surgical procedure with the consequent positive effect on patients and relatives reducing the rate of refusal to sign the informed consent form $(33,34)$.

The range of clinical indications of $3 \mathrm{D}$ printing in the field of neurosurgery is almost endless (32). Consensus guidelines with recommendations for image acquisition protocols and image data preparation, manipulation and printing have been carried out by expert panels, which can be used as a reference (35). Some clinical groups are including in the physical models the information provided by DTI and fMRI on advanced 3D physical models for a better delimitation of white matter tracts and eloquent cortical areas (36). Presurgical assessments of craniosynostosis or skull defect implants are also being addressed by using $3 \mathrm{D}$ printing techniques, based on CT 

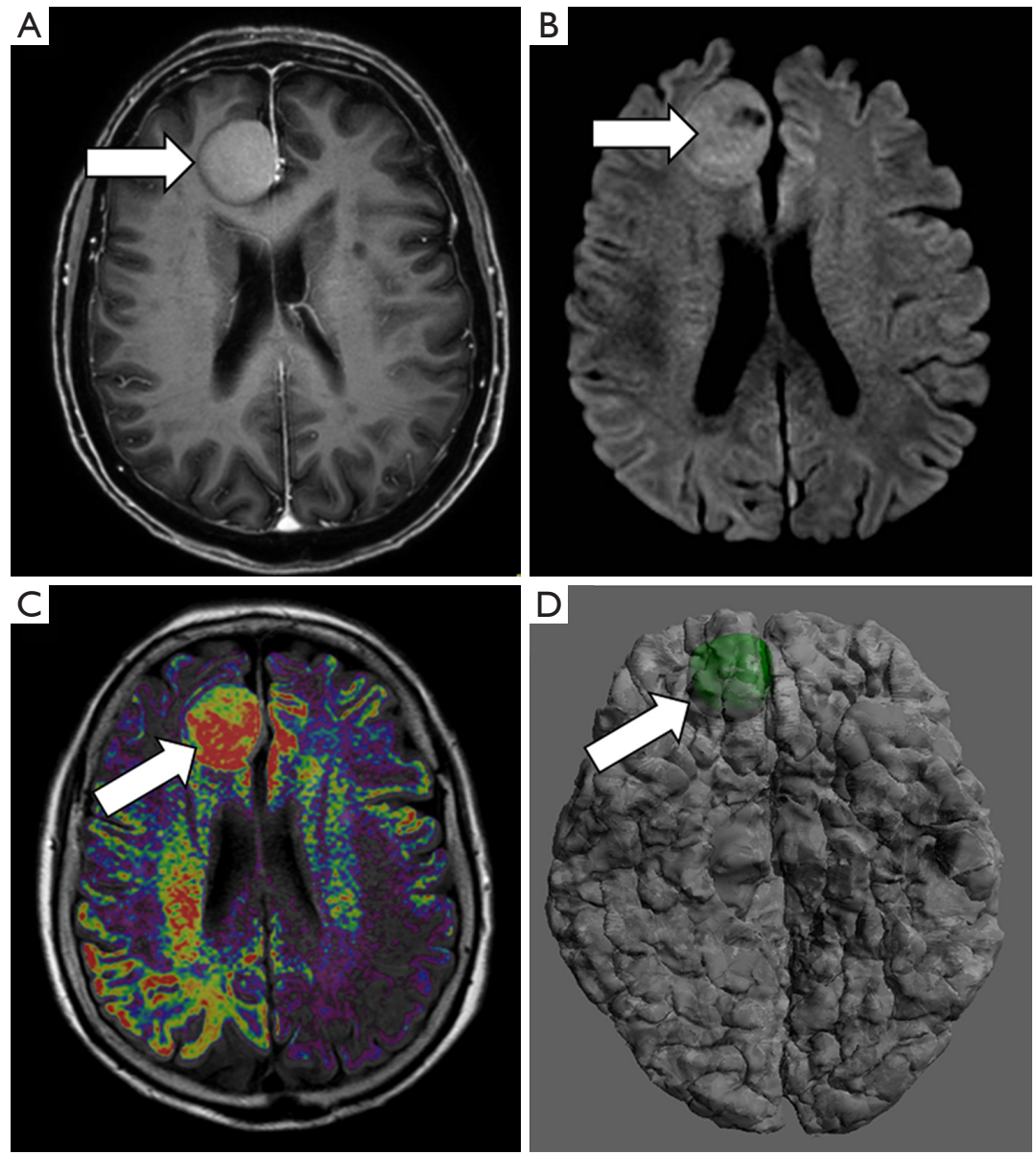

Figure 2 Meningioma presurgical evaluation. An 81-year-old female with right frontal lobe meningioma. (A) Axial gadolinium-enhanced T1w image shows a large right parasagittal frontal extra-axial lesion consistent with meningioma (arrow). (B) Axial b 1,000 s/mm ${ }^{2} \mathrm{DWI}$ identifies severe restriction of water diffusion within the meningioma which suggests hypercellular lesion (arrow). (C) The fusion of functional information from DWI and morphological information form T1W images demonstrates proper correlation identifying areas of higher hypercellularity within the posterior aspect of the meningioma (arrow). (D) 3D model merging anatomical information from morphological MRI and functional information from DWI allows the evaluation of those areas with a higher restriction of water diffusion within meningioma inside the whole brain anatomy (arrow).

segmentations $(37,38)$. Neurovascular applications are mainly focused on the presurgical planning of vascular malformations and aneurysms, including hemodynamic simulations, for the planification of both interventional endovascular or open surgery approaches $(32,39,40)$. Spine surgery may also benefit from the use of $3 \mathrm{D}$ printed models for the evaluation of scoliosis or other types of congenital spine malformations as well as for complex vertebral bone lesions, especially those which involve the spinal canal and/ or cord (18).

To the best of our knowledge, scarce publications have addressed the potential usefulness of hybrid CT and MRI
$3 \mathrm{D}$ modeling and printing in neurosurgery, or even less the addition of information from advanced functional MRI techniques to this process. The emergence of affordable 3D printing technologies has led to a significant increase in the use of this approach in clinical practice. Nevertheless, not all printers are capable of printing multi-color or multimaterial models $(41,42)$. The most accessible printers are limited to a single color and material per print. Another current limitation of $3 \mathrm{D}$ printing is the time required to create the model. The printing of a complex model, including the post-processing time that each technology requires, can take up to 4 days per model. These times can 


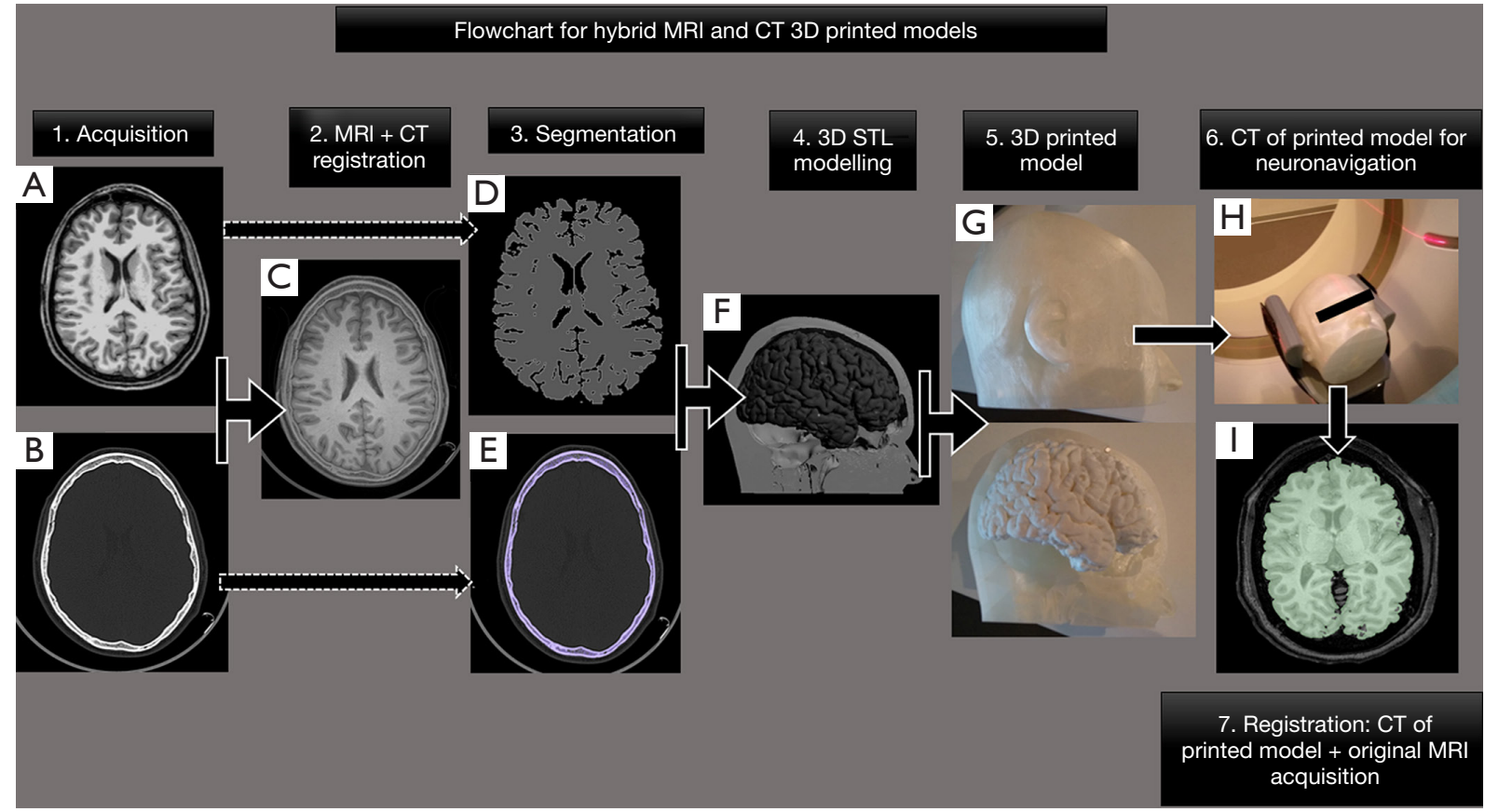

Figure 3 Flowchart for hybrid MRI and CT 3D printed model for epilepsy pre-surgical planning. A 25-year-old male with a drug-resistant epilepsy candidate for focal thermocoagulative therapy. Neurosurgeons ask for the possibility of obtaining a 3D printed model of the whole patient head including soft tissues, skull, and brain for proper planning of electrodes and guides positioning. Information from both (A) axial 3D-T1W and (B) CT images were used for (C) registration and segmentation of (D) gray matter and (E) bone. (F) Hybrid 3D model in STL format was generated and sent to 3D print obtaining a $(\mathrm{G}) 3 \mathrm{D}$ printed model which included information of both the brain, soft tissues, and skull. $(\mathrm{H})$ The printed brain model has undergone a CT scan which perfectly fitted with its source image form MRI (I). 3D printed model enables neurosurgeons to plan electrodes positioning and surgical entry points before surgery.

be assumed for elective neurosurgical procedures as most of neuro-oncology or congenital malformations cases, but it makes it less practical for urgent CNS or spine pathologies, such as brain or spinal trauma.

\section{Technical tips for hybrid CT and MRI 3D printing}

Image acquisition aside, the process of creating a $3 \mathrm{D}$ printed model from multimodality images requires facing 4 main tasks: image registration, segmentation, 3D modeling and $3 \mathrm{D}$ printing following a specific flowchart summarized in Figure 3.

Image registration is the process of superimposing and aligning two or more images so that they share a common space. This process allows combining information from different imaging series or acquisitions, including both anatomical and functional information, enabling the creation of more complex $3 \mathrm{D}$ models since anatomical structures and lesions can be segmented in different images.
In neurosurgery, this allows the extraction of bone tissue and vessels from CT scans, gray and white matter volumes from 3D T1-weighted MRI series, white matter tracts from DTI, or adding other type of physiopathological information provided from advanced MRI sequences and to combine all this information in a single $3 \mathrm{D}$ model. To register information from CT and MRI, the algorithms based on mutual information $(43,44)$ have proven their effectiveness for CNS evaluation in the literature (45-47). Regarding neurosurgical applications, rigid registration approaches based on that mutual information have been successfully used for the overlay of high-resolution T2weighted images and CT series of the head area $(48,49)$. One of the most used solutions to implement this type of registration is the Elastix Framework ${ }^{\odot}$ (50), which is open-source software that allows to carry out both rigid and deformable registration using a variety of metrics, including normalized mutual information. There are other commercial solutions, such as Philips IntelliSpace Portal ${ }^{\odot}$ 
(v10.1, 15 June 2018, Koninklijke Philips Electronics N.V.), that make rigid registrations and provide interactive tools to fine-tune them.

After the registration process, each of the tissues (bone, vessels, lesions, white matter tracts or brain volumes) to be represented in the $3 \mathrm{D}$ model can be segmented into a different image series, or even in more than one image acquisition, to generate only one of the $3 \mathrm{D}$ models parts. After this step, the previous registration process enables joining all the different segmentation results to build the final model. Nevertheless, not all the tissues can be segmented using the same strategy. A valid algorithm for bone segmentation from a CT will surely perform inadequately in the gray matter segmentation using a $3 \mathrm{D}$ T1-weighted MRI series; hence each segmentation goal requires a different approach.

The segmentation of bone tissue, skull, and spine from CT images is a process that has been widely addressed in literature (51), and that can be solved using intensity-based methods. The segmentation of vessels from CT angiography can be better performed using level sets (52). Gray and white matter volumes can be automatically extracted from non-contrast 3D T1-weighted MRI acquisitions using FreeSurfer $^{\odot}$ or Brainsuite ${ }^{\Theta}(53,54)$. These softwares also allow to automatically parcellate surfaces to identify different areas. This parcellation permits the generation of models of specific surface areas, or even, colored models delimiting each area. The automatic segmentation of the spinal cord from the T1-weighted or T2-weighted MRI series can be achieved by using the Spinal Cord Toolbox (55). Brain tumors can be automatically segmented from multiparametric MRI using potential field-based methods (56) or convolutional neural networks (CNN) (57). However, most of the time brain tumors are finally manually segmented by neuroradiologists, due to both the simplicity of the segmentation of this type of tissues using semiautomatic tools and the need for a thorough review of the segmentation results by the radiologist

Regarding the combination of functional and anatomical information in additive manufacturing, it is almost an unexplored field of research. White matter tracts extracted from DTI can be included in the 3D model by previously registering DTI to anatomical images, most commonly using CT or 3D T1-weighted MRI series being as reference $(58,59)$. Using $3 \mathrm{D}$ Slicer $^{\odot}$ (version 4.10.1), fiber tracts can be generated from DTI and exported as 3D models (60). This procedure enables the combination of fiber tracts together with the skull, brain surface, lesions or vessels in the same $3 \mathrm{D}$ model. Another way of incorporating functional information in a $3 \mathrm{D}$ model is to enhance lesions previously segmented on an fMRI study (61) (Figure 4). An approach that takes better advantage of the information from the functional series is to display fMRI activation maps on top of the brain surface in the $3 \mathrm{D}$ printed model. After registering $3 \mathrm{D}$ T1-weighted and $\mathrm{FMRI}$ images, activation maps can be derived from fMRI and then projected on the brain surface by using FreeSurfer ${ }^{\odot}$ (62).

Once tissues have been segmented and 3D models have been generated, they need to be processed to obtain printable 3D models. The most extended printable format is STL (STereoLithography), although there are many other types of files, which allows the implementation of different textures or colors, and must be chosen according to the final application of the physical model. The printable model encloses a $3 \mathrm{D}$ surface defined by several triangular facets to fit these surfaces, forming a truly triangle mesh, which is formed by multiple edges, faces, and connecting points. Firstly, it is necessary to check that the $3 \mathrm{D}$ model is closed, it does not contain duplicated faces and its normal vectors are properly oriented. For example, if boundary edges are present, the STL may show areas without information (holes) avoiding a complete closure of its surface (63). Then, all the different parts that compose the model must be artificially connected to avoid that spatial references are lost after printing, preserving the original design. Additionally, in some cases, artificial sections are created to create a better visualization of the inner parts of the $3 \mathrm{D}$ models. On the other hand, it is important to control the error that is made when generating and processing $3 \mathrm{D}$ models. For that purpose, model contours can be superimposed on the CT or MRI image to visually compare model boundaries. In the planning of brain surgery with neuronavigation, a CT scan of the physical model can be performed on the printed model to optimize the procedure. This new CT can be compared to the original MRI acquisition of the real patient by previously registering them to assess the error committed during the entire $3 \mathrm{D}$ printing process (Figure 3). Finally, the 3D model is printed. The printing technology is also chosen according to the colors or materials required by the model and the size of the smallest details that must be printed. Hybrid CT and MRI 3D models usually contain different tissues; therefore, they require a multicolor technology such as ColorJet. In the case each tissue needs to have different material properties, PolyJet or MultiJet are preferred for printing, especially the models generated from fMRI data may 

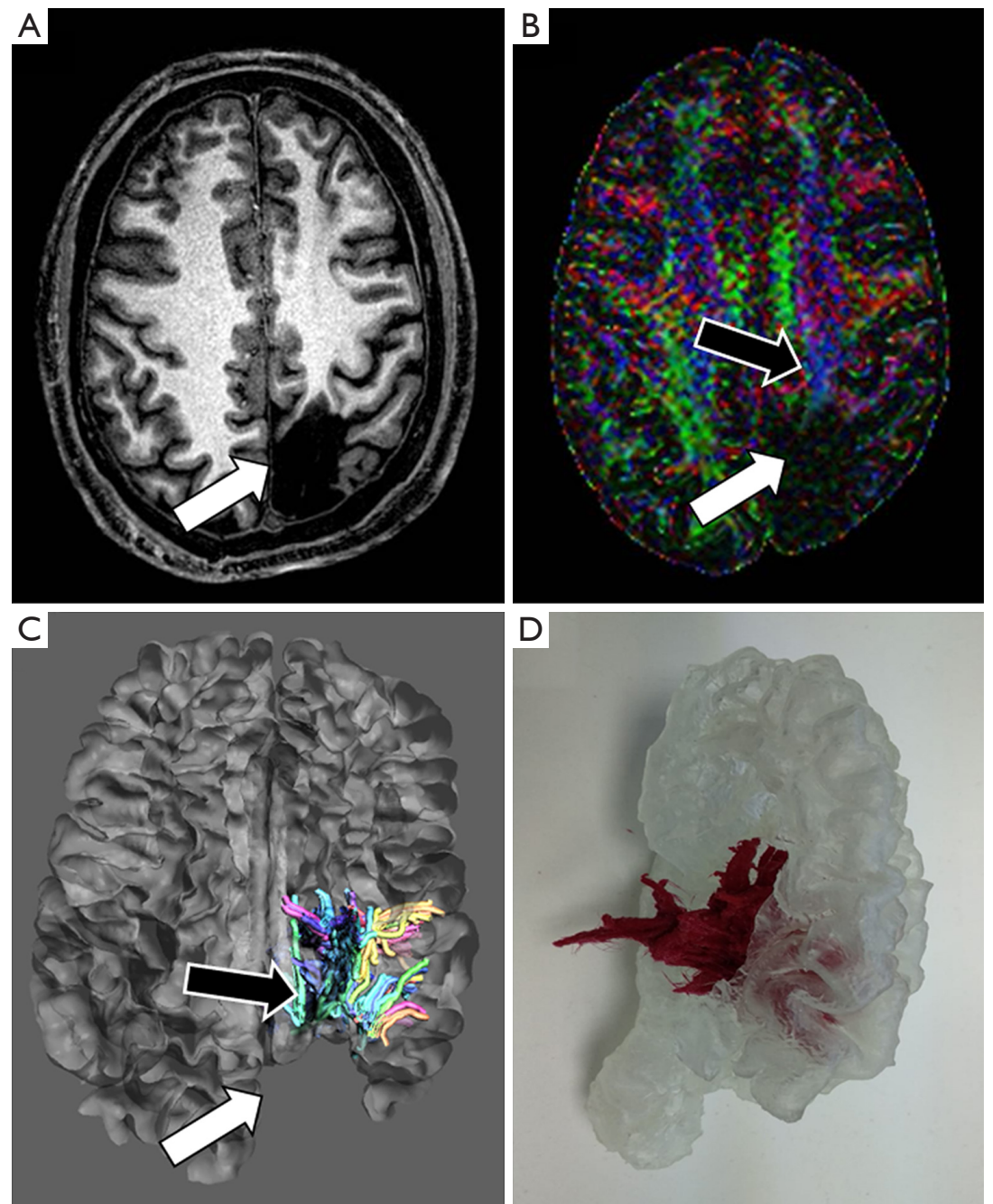

Figure 4 Presurgical planning of focal epilepsy. A 37-year-old male, with a history of previous surgical left parieto-occipital resection 20 years ago, refers to occipital lobe seizures. Neurologists asked for a presurgical study to minimize the damage of both optical and corticospinal pathways. (A) Axial T1-weighted image shows a large left parieto-occipital area of encephalomalacia (arrow). (B) Color-coded Fraction Anisotropy map, generated from DTI acquisition identifies the involvement of posterior tracts of left corona radiata (white arrow) with apparent preservation of corticospinal tract (black arrow). (C) The fusion of morphological MRI and DTI images allows evaluating the relationships between white matter tracts (black arrow) and post-surgical cavity (white arrow). (D) 3D printed model enables neurosurgeons to address physically the location of white matter tracts for a more secure surgical planning.

benefit from using these types of printers (42). Otherwise, each tissue can be printed separately, and then all the tissues can be joined manually to build the model. At the expense of a higher cost per model, multi-color and multimaterial printers require less time to create models, since the printing time is usually shorter and complex models can be created in a single print, reducing post-processing time (42). See Table 1 for further details on currently available printing technologies.

\section{Clinical applications of hybrid CT and MRI 3D models}

The merging of information derived from CT and MRI has not been fully exploited in neurosurgery and may help to resolve several specific problems in the workflow of $3 \mathrm{D}$ modeling and printing. In most neurosurgical patients, there is a need for accurate identification of bone at the skull or spine to establish basic anatomical references for surgical 

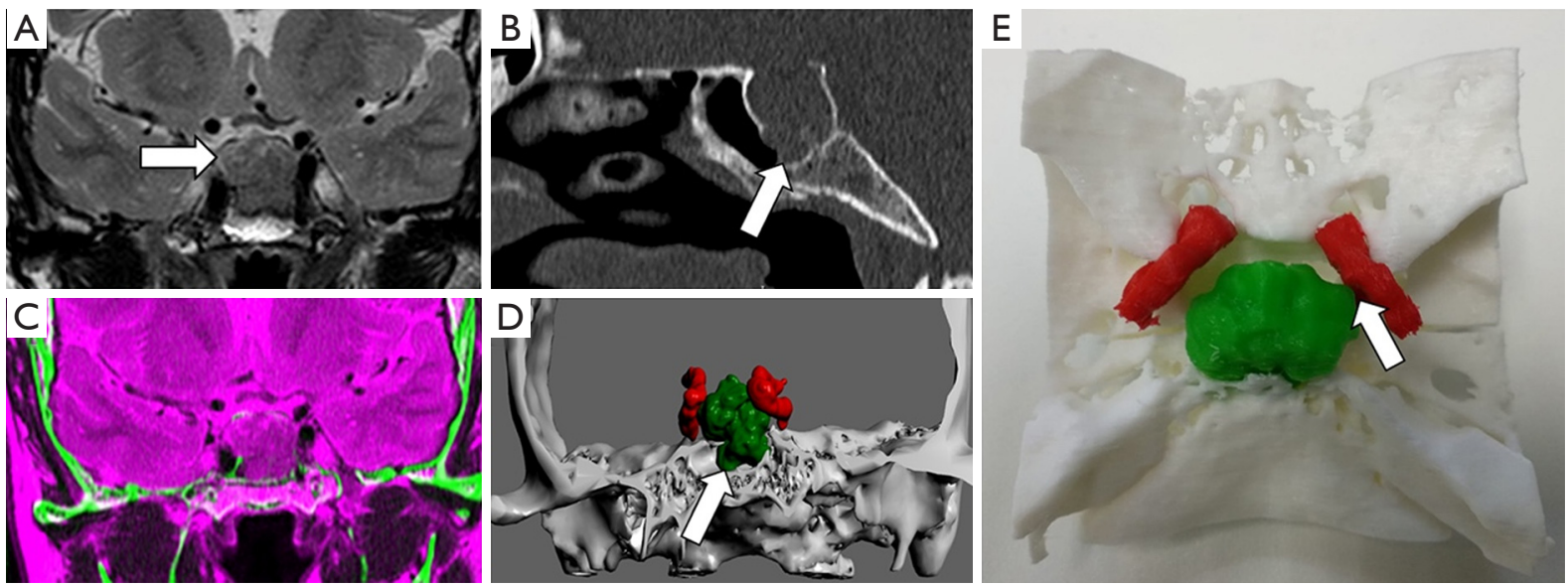

Figure 5 Presurgical planning of hypophyseal macroadenoma. A 54-year-old female that undergone MRI for headache and vision loss. (A) Coronal T2 TSE shows a large sellar mass that seems to invade sphenoidal sinus (arrow). (B) Sagittal reconstruction of CT confirms the presence of an enlarged sella turcica with disruption of its floor (arrow). (C) Registration of information of skull base structures from CT (green) and data from MRI (magenta) enables an adequate tissue contrast for adenoma and even internal carotids segmentation. (D) Coronal projection of the $3 \mathrm{D}$ combined model allows neurosurgeons to properly evaluate the relationship of the hypophyseal mass (codded in green) with sphenoidal sinus, cavernous sinus and even both internal carotids (codded in red), demonstrating disruption of sella turcica floor (arrow). (E) Zenithal view of 3D printed model shows proper correlation with radiological images and $3 \mathrm{D}$ virtual model demonstrating contact between macroadenoma and left internal carotid (white arrow).

planning. For this aim, data provided by CT is optimal, as it enables the best way to discriminate between bone and soft tissues in a faster and accurate manner. However, softtissue contrast, especially between gray or white matter and brain lesions is suboptimal when CT information is used to perform segmentation of CNS structures. Similarly, it is hard to discriminate between cerebrospinal fluid (CSF) and spinal cord using only the CT data. On the other hand, while MRI provides a better contrast of soft tissues or brain parenchyma, and nicely separates the spinal cord from CSF, especially using $\mathrm{T} 2$-weighted sequences. However, the ability of MRI to evaluate skull, vertebral bodies and bone cortex is still limited compared with CT (17).

Complex surgical procedures, which involve both soft tissues, such as brain tumors, intracranial vessels or even subcutaneous fat pad, and skull base or calvarium, may benefit from the use of this combined approach. Posterior fossa lesions, hypophyseal macroadenomas or tumors in the vicinity of the sphenoidal plane or cribriform plate may be printed using this hybrid approach to facilitate surgical planning and minimize possible complications (64) (Figure 5). 3D printing of craniovertebral junction has also demonstrated to be useful for addressing this complex area, permitting the conjoined assessment of both bony structures (clivus, odontoid process, atlas, and foramen magnum), and brainstem/medulla (65). In the particular case of brain vascular malformations and aneurysms, information from 3D-CT angiography (CTA), which provides high contrast between vessels and brain parenchyma, results in an optimal vascular definition for 3D modeling (66). This data can be complemented with MR-angiography (MRA) approaches, such as time-of-flight (TOF), contrast-enhanced 3D MRA and more recently 4D time-resolved MRA acquisitions, which provide a wide range of different vascular phases which allow neuroradiologists and neurosurgeons to have all the information necessary for a comprehensive vascular assessment avoiding the use of ionizing radiation $(67,68)$. If data from both angiographic imaging modalities are combined, $3 \mathrm{D}$ models can be printed integrating truly realistic anatomical and hemodynamic information about vascular malformation behavior (Figure 6).

$3 \mathrm{D}$ printing models have demonstrated their usefulness in the presurgical assessment of the spine and spinal cord lesions (18). Due to the complexity of this anatomical region, the use of hybrid CT and MRI 3D printed models may allow neurosurgeons to have at their disposal an accurate physical representation that permits a safer guiding of bone or spinal cord biopsies, or the improved planning of 

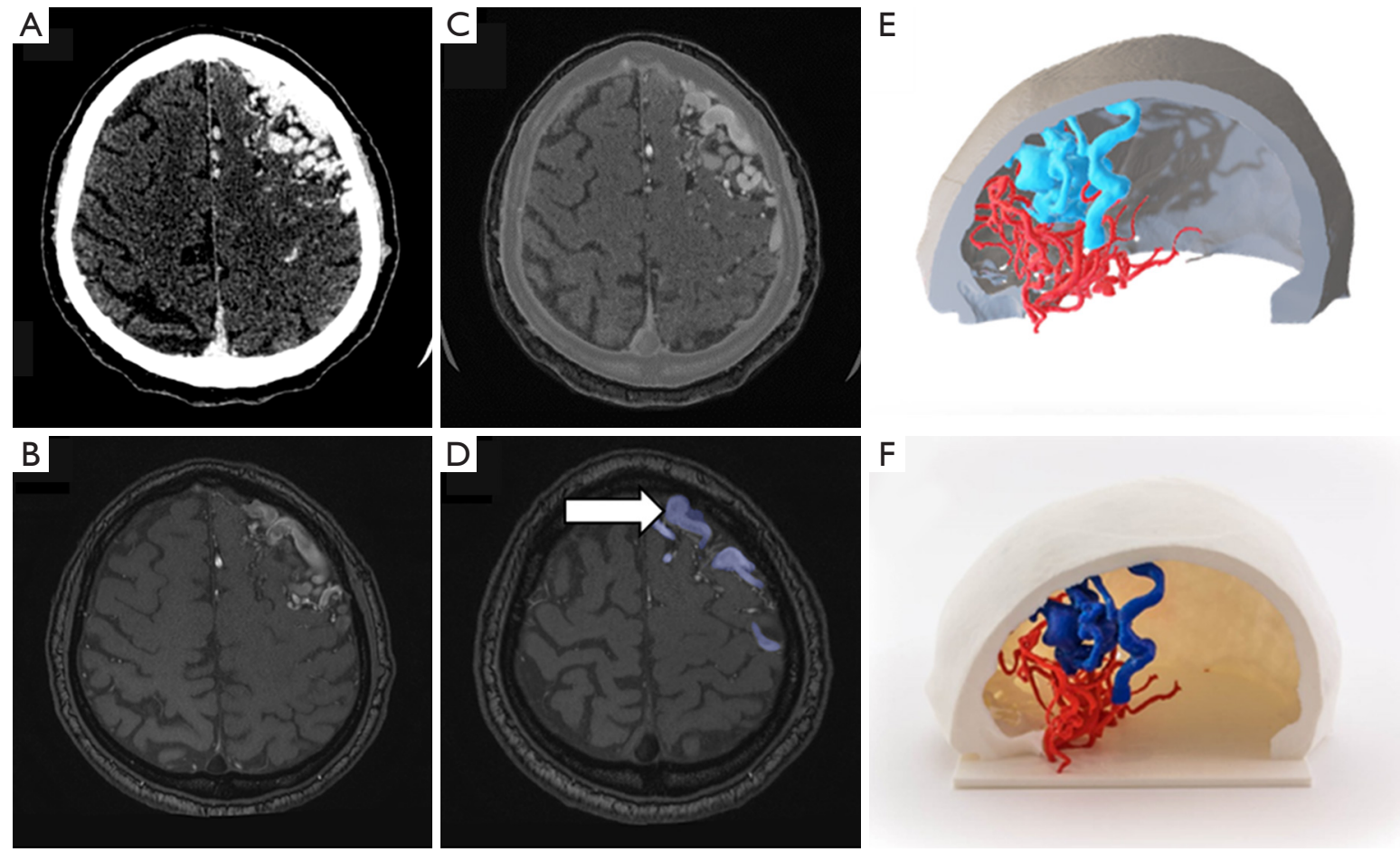

Figure 6 Brain arteriovenous malformation assessment. A 35-year-old male with left frontal AVM that undergone both CT-angiography and MRI-angiography studies. Information obtained from (A) CT was used for the skull and both arteries and veins segmentation (B). (C) MR-angiography using time of flight (TOF) technique with arterial velocity codification allows accurately segment high flow veins (arrows at Figure 6D) that compose the AVM as well as differentiate them from the brain parenchyma and skull. Combined 3D modeling (E) and 3D printed models $(\mathrm{F})$ provide neurosurgeons and interventional radiologists a comprehensive assessment of AVM and its relationship with the skull.

surgical procedures $(61,69)$ (Figure 7). Moreover, it will be recommended to use this type of hybrid approach in the $3 \mathrm{D}$ design of surgical guides for proper identification of spinal cord or other soft tissue structures. Thus, the risk of spinal cord damage will be minimized thanks to the inclusion of information about its relationship with surgical guides and possible target (focal lesion biopsy or external device implantation) (70).

\section{Clinical applications of hybrid morphological and functional MRI 3D printing}

The combined use of information provided from conventional anatomical and advanced MRI sequences is very promising for applications of additive manufacturing in neurosurgery. Most of the reported studies are focused on the integration of data from $\mathrm{fMRI}$ into the $3 \mathrm{D}$ printed model (36). This approach enables to visualize the activation of those eloquent areas, facilitating the spatial relationship between normal brain gyrus and tumors. In the same line, the inclusion in physical models of the information from DTI studies, especially from tractographic reconstructions, has also been proposed as a potential advantage for oncological surgical planning allowing to visualize more realistically their relationship with brain lesions $(58,59)$. These 3D printed models may help neurosurgeons to evaluate all the possible surgical approaches and types of resections to preserve patient functions and reduce tumoral bulk, as much as possible (61). In the field of the electrodes positioning for brain stimulation or radiofrequency techniques, some authors have proposed the use of hybrid CT and MRI models to confirm the correct location of electrodes in deep brain stimulation such as in Parkinson disease (48).

DWI and PWI are advanced sequences which have been included as part of routine protocols for brain tumor assessment by neuroradiologists. However, these sequences have been uncommonly incorporated into $3 \mathrm{D}$ printed models. 

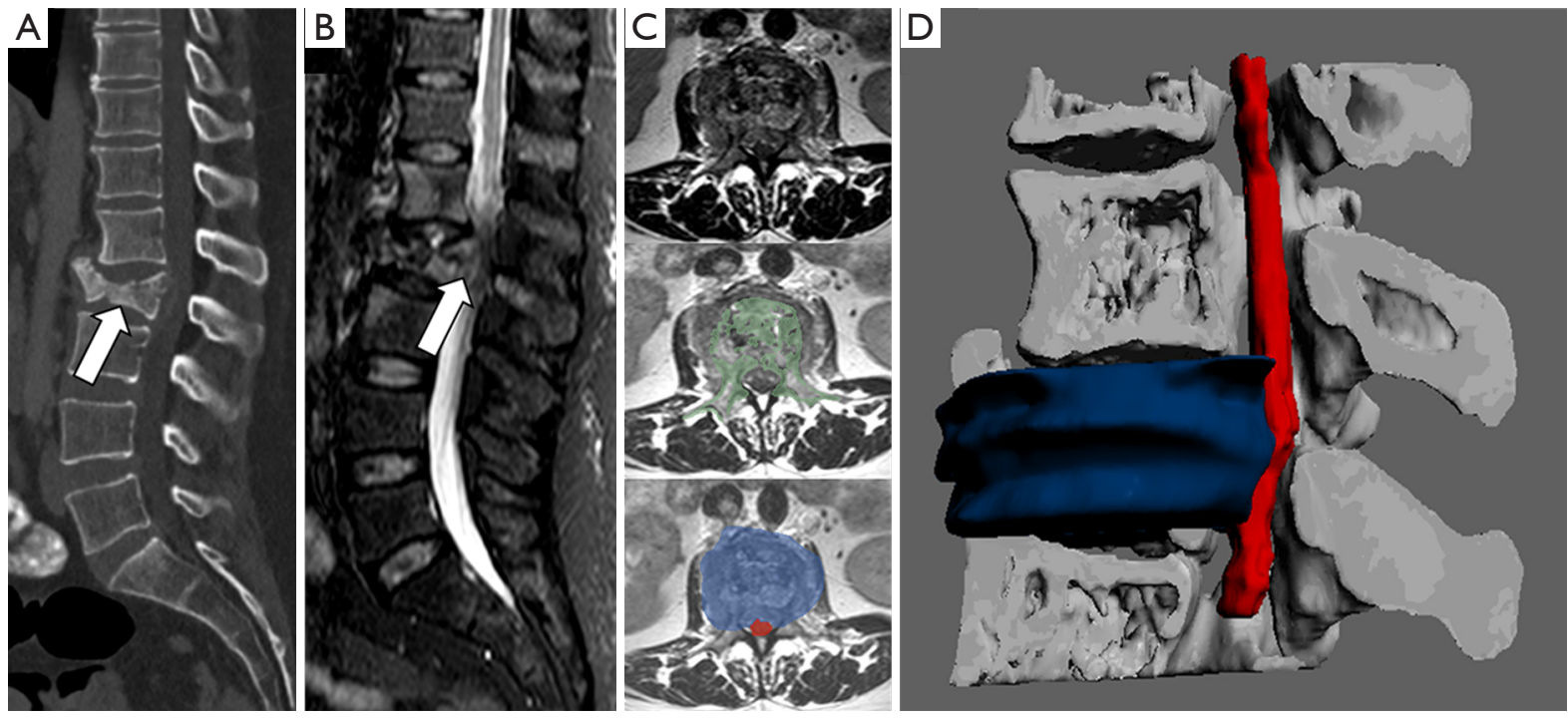

Figure 7 Spine and spinal cord evaluation. A 42-year-old female with a history of breast cancer is evaluated in an emergency room for lumbar pain and lower limb paresis. (A) Sagittal reconstruction of CT shows L2 vertebral body collapse which seems to invade the spinal canal (arrow). (B) Sagittal STIR sequence confirms the existence of a metastatic fracture with soft tissue component that invades spinal canal conditioning severe stenosis. (C) Registration of information form axial T2-TSE with CT allows to segment vertebral bone (codded in green) and also soft tissue mass (codded in blue) and spinal cord (codded in red). (D) The 3D model enables neurosurgeons to visualize in a single view all the information form both CT and MRI and identify how the soft tissue component (codded in blue) invades the spinal canal and displaces the spinal cord.

Information about brain tumor cellularity provided by DWI has a recognize value for tumor grading and detection of progression after chemo-radio or even surgical therapy (10). PWI is also able to determine the presence of areas of neoangiogenesis within brain lesions (71). Information about lesions vascularization can also be obtained from noncontrast MRI sequences, as arterial spin labelling (ASL). ASL has demonstrated similar accuracy than conventional contrast-enhanced PWI studies for CNS lesion detection and characterization (72). Most commonly, presurgical MRI scans for neuronavigation purposes only include non-contrast anatomical sequences, the addition of noncontrast advanced sequences such as DWI or ASL can facilitate the inclusion of this physiologic information into the $3 \mathrm{D}$ models (Figure 8). In this manner, the neurosurgeons can potentially have all the information to perform a more targeted biopsy in a single physical model, focused on the area of higher biological aggressiveness, as identified on DWI or ASL sequences. Also, these 3D printed models can facilitate a more efficient surgical resection of both primary brain tumor or suspicious tumor recurrence, due to a better knowledge of their local extension and tumor heterogeneity.

\section{Conclusions}

The use of 3D modeling, and particularly 3D printing, is demonstrating several advantages in the field of neurosurgery being its most extended use the surgical planning. Physical models have shown to improve communication between specialists and physicians and patients, and their usefulness training neurosurgeons. Nevertheless, there are specific clinical scenarios in which conventional 3D printing approaches are not enough for obtaining a sufficiently specific anatomical or physiopathological detail of the structure or pathology represented. In these cases, the use of hybrid CT and MRI information, including advanced MRI sequences, may help to integrate the different sources of information in one single $3 \mathrm{D}$ printed model. This type of hybrid $3 \mathrm{D}$ modeling requires specific technical adjustments, segmentation and registering software, and even more, sophisticated 3D printers and materials. The following challenge is to demonstrate the potential positive impact of these advanced $3 \mathrm{D}$ printed models in neurosurgical procedures and patient outcomes with multicenter trials. 

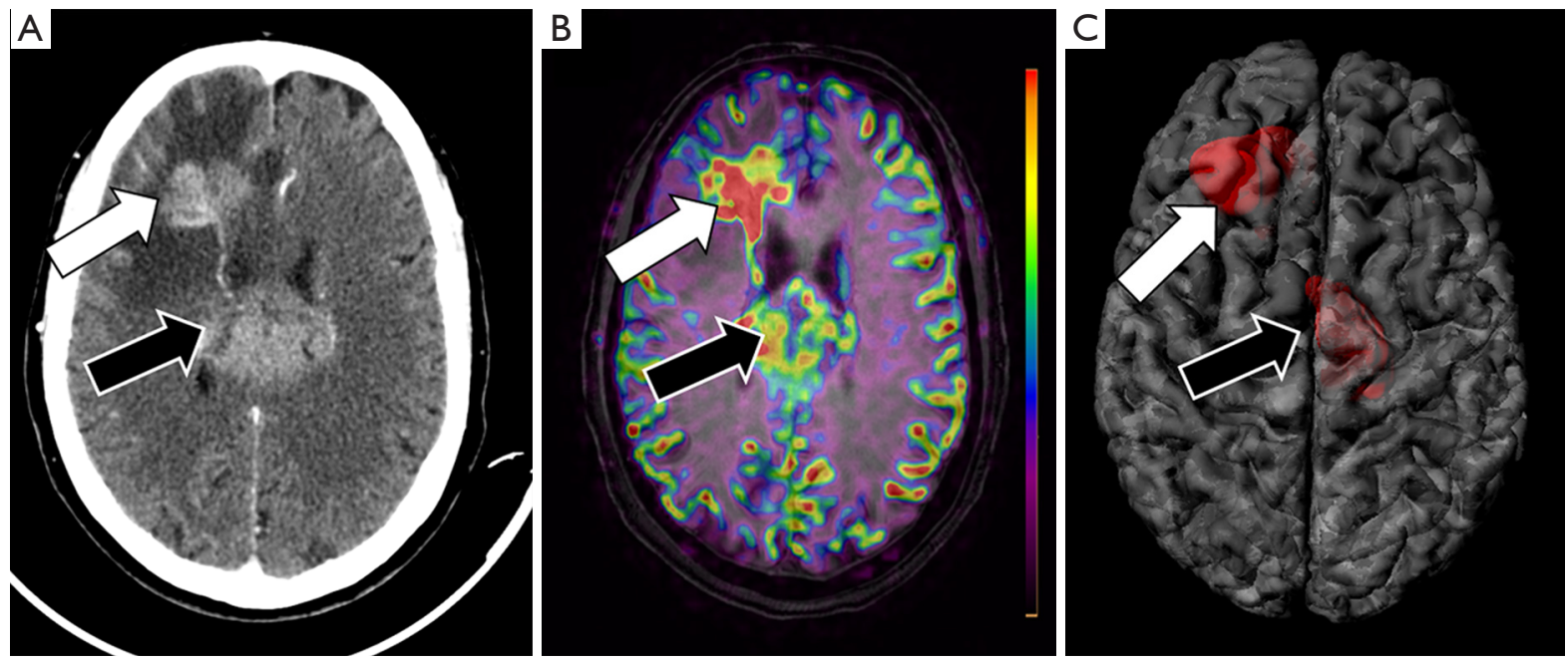

Figure 8 Multimodality assessment of a CNS tumor. A 73-year-old female with changes in behavior and cognitive impairment undergone (A) contrast-enhanced CT that shows two large hyperenancing masses at right frontal lobe (white arrow) and within the corpus callosum (black arrow). (B) MRI was performed using ASL technique for assessment of tumor blood flow before guide biopsy to avoid the administration of gadolinium. The fusion of morphological MRI sequence (FLAIR) and functional MRI sequence (ASL) allows neuroradiologists to characterize the right frontal lesion as a potentially higher grade compared with the corpus callosum lesion as the former shows higher blood flow values (white arrow) compared with the latter (black arrow). (C) The register of morphological MRI and functional (ASL) MRI data allows the generation of a $3 \mathrm{D}$ model in a single file with all the information needed for a proper planification of brain biopsy.

\section{Acknowledgments}

Camilo Riascos for the edition and revision of the final manuscript draft.

Funding: This work has been partially funded by the Ministry of Science, Innovation and Universities of the Government of Spain, through the Torres Quevedo program under grant PTQ-16-08419.

\section{Footnote}

Conflicts of Interest: The authors have no conflicts of interest to declare.

Ethical Statement: The authors are accountable for all aspects of the work in ensuring that questions related to the accuracy or integrity of any part of the work are appropriately investigated and resolved.

\section{References}

1. Liaw CY, Guvendiren M. Current and emerging applications of 3D printing in medicine. Biofabrication
2017;9:024102.

2. Rengier F, Mehndiratta A, Von Tengg-Kobligk H, et al. 3D printing based on imaging data: Review of medical applications. Int J Comput Assist Radiol Surg 2010;5:335-41.

3. Wadhwa A, Bhardwaj A, Singh Verma V. A review on brain tumor segmentation of MRI images. Magn Reson Imaging 2019;61:247-59.

4. Hoang D, Perrault D, Stevanovic M, et al. Surgical applications of three-dimensional printing: a review of the current literature \& how to get started. Ann Transl Med 2016;4:456.

5. Waran V, Pancharatnam D, Thambinayagam HC, et al. The utilization of cranial models created using rapid prototyping techniques in the development of models for navigation training. J Neurol Surg A Cent Eur Neurosurg 2014;75:12-5.

6. Guo F, Dai J, Zhang J, et al. Individualized 3D printing navigation template for pedicle screw fixation in upper cervical spine. PLoS One 2017;12:e171509.

7. Castle M, Rivero M, Marquez J. Primary Ewing's sarcoma of the skull: Radical resection and immediate cranioplasty after chemotherapy. Childs Nerv Syst 2013;29:303-6.

8. McMenamin PG, Quayle MR, McHenry CR, et al. The 
production of anatomical teaching resources using threedimensional (3D) printing technology. Anat Sci Educ 2014;7:479-86.

9. Grillo FW, Souza VH, Matsuda RH, et al. Patient-specific neurosurgical phantom: assessment of visual quality, accuracy, and scaling effects. 3D Print Med 2018;4:3

10. Brandão LA, Shiroishi MS, Law M. Brain Tumors: A Multimodality Approach with Diffusion-Weighted Imaging, Diffusion Tensor Imaging, Magnetic Resonance Spectroscopy, Dynamic Susceptibility Contrast and Dynamic Contrast-Enhanced Magnetic Resonance Imaging. Magn Reson Imaging Clin N Am 2013;21:199-239.

11. Wang Y, Li C, Zhu T, et al. Multimodal brain tumor image segmentation using WRN-PPNet. Comput Med Imaging Graph 2019;75:56-65.

12. Bohl MA, Morgan CD, Mooney MA, et al. Biomechanical Testing of a 3D-printed L5 Vertebral Body Model. Cureus 2019;11:e3893.

13. Clifton W, Nottmeier E, Damon A, et al. A Feasibility Study for the Production of Three-dimensionalprinted Spine Models Using Simultaneously Extruded Thermoplastic Polymers. Cureus 2019;11:e4440.

14. Chen H, Zhang J, Li X, et al. Multi-level customized $3 \mathrm{D}$ printing for autogenous implants in skull tissue engineering. Biofabrication 2019;11:045007

15. Chia $\mathrm{HN}, \mathrm{Wu} \mathrm{BM}$. Recent advances in $3 \mathrm{D}$ printing of biomaterials. J Biol Eng 2015;9:4.

16. Mandrycky C, Wang Z, Kim K, et al. 3D bioprinting for engineering complex tissues. Biotechnol Adv 2016;34:422-34.

17. Naftulin JS, Kimchi EY, Cash SS. Streamlined, inexpensive $3 \mathrm{D}$ printing of the brain and skull. PLoS One 2015;10:e0136198.

18. Wilcox B, Mobbs RJ, Wu AM, et al. Systematic review of 3D printing in spinal surgery: the current state of play. $\mathrm{J}$ Spine Surg 2017;3:433-43.

19. Shen Z, Yao Y, Xie Y, et al. The process of 3D printed skull models for anatomy education. Comput Assist Surg (Abingdon) 2019;24d:121-30.

20. Mashiko T, Konno T, Kaneko N, et al. Training in Brain Retraction Using a Self-Made Three-Dimensional Model. World Neurosurg 2015;84:585-90.

21. Izatt MT, Thorpe PLPJ, Thompson RG, et al. The use of physical biomodelling in complex spinal surgery. Eur Spine J 2007;16:1507-18.

22. Pacione D, Tanweer O, Berman P, et al. The utility of a multimaterial 3D printed model for surgical planning of complex deformity of the skull base and craniovertebral junction. J Neurosurg 2016;125:1194-7.

23. Choy WJ, Parr WCH, Phan K, et al. 3-Dimensional Printing for Anterior Cervical Surgery: a Review. J Spine Surg 2018;4:757-69.

24. Sandler AL, Tepper OM, Goodrich JT, et al. Use of a customized 3D "basket" to create a solitary split-thickness cranial graft from numerous split fragments in an infant. J Neurosurg Pediatr 2014;14:196-9.

25. Vakharia VN, Vakharia NN, Hill CS. Review of 3-Dimensional Printing on Cranial Neurosurgery Simulation Training. World Neurosurgery 2016;88:188-98.

26. Tai BL, Rooney D, Stephenson F, et al. Development of a 3D-printed external ventricular drain placement simulator: technical note. J Neurosurg 2015;123:1070-6.

27. Chen J, Li N, He D, et al. 3-D printing for constructing the burr hole ring of lead fixation device in deep brain stimulation. J Clin Neurosci 2018;58:229-33.

28. Gao F, Wang Q, Liu C, et al. Individualized 3D printed model-assisted posterior screw fixation for the treatment of craniovertebral junction abnormality: a retrospective study. J Neurosurg Spine 2017;27:29-34.

29. Hassan MN, Yassin MA, Suliman S, et al. The bone regeneration capacity of $3 \mathrm{D}$-printed templates in calvarial defect models: A systematic review and meta-analysis. Acta Biomater 2019;91:1-23.

30. Cecchinato R, Berjano P, Zerbi A, et al. Pedicle screw insertion with patient-specific 3D-printed guides based on low-dose CT scan is more accurate than free-hand technique in spine deformity patients: a prospective, randomized clinical trial. Eur Spine J 2019;28:1712-23.

31. Deng T, Jiang M, Lei Q, et al. The accuracy and the safety of individualized $3 \mathrm{D}$ printing screws insertion templates for cervical screw insertion. Comput Assist Surg (Abingdon) 2016;21:143-9.

32. Randazzo M, Pisapia J, Singh N, et al. 3D printing in neurosurgery: A systematic review. Surg Neurol Int 2016;7:S801-9.

33. Liew Y, Beveridge E, Demetriades AK, et al. 3D printing of patient-specific anatomy: A tool to improve patient consent and enhance imaging interpretation by trainees. Br J Neurosurg 2015;29:712-4.

34. Zhuang YD, Zhou MC, Liu SC, et al. Effectiveness of personalized 3D printed models for patient education in degenerative lumbar disease. Patient Educ Couns 2019;102:1875-81.

35. Chepelev L, Wake N, Ryan J, et al. Radiological Society of North America (RSNA) 3D printing Special Interest 
Group (SIG): guidelines for medical 3D printing and appropriateness for clinical scenarios. 3D Print Med 2018;4:11.

36. Spottiswoode BS, Van Den Heever DJ, Chang Y, et al. Preoperative three-dimensional model creation of magnetic resonance brain images as a tool to assist neurosurgical planning. Stereotact Funct Neurosurg 2013;91:162-9.

37. Morales-Gómez JA, Garcia-Estrada E, Leos-Bortoni JE, et al. Cranioplasty with a low-cost customized polymethylmethacrylate implant using a desktop 3D printer. J Neurosurg 2018. [Epub ahead of print].

38. Fiaschi P, Pavanello M, Imperato A, et al. Surgical results of cranioplasty with a polymethylmethacrylate customized cranial implant in pediatric patients: a single-center experience. J Neurosurg Pediatr 2016;17:705-10.

39. Russ M, O'hara R, Nagesh SVS, et al. Treatment Planning for Image-Guided Neuro-Vascular Interventions Using Patient-Specific 3D Printed Phantoms. Proc SPIE Int Soc Opt Eng 2016;9417:941726.

40. Sullivan S, Aguilar-Salinas P, Santos R, et al. Threedimensional printing and neuroendovascular simulation for the treatment of a pediatric intracranial aneurysm: case report. J Neurosurg Pediatr 2018;22:672-7.

41. Mitsouras D, Liacouras P, Imanzadeh A, et al. Medical 3D Printing for the Radiologist. Radiographics 2015;35:1965-88.

42. Kim GB, Lee S, Kim H, et al. Three-dimensional printing: Basic principles and applications in medicine and radiology. Korean J Radiol 2016;17:182-97.

43. Pluim JPW, Maintz JBA, Viergever MA. Mutualinformation-based registration of medical images: a survey. IEEE Trans Med Imaging 2003;22:986-1004.

44. Oliveira FPM, Tavares JMRS. Medical image registration: A review. Comput Methods Biomech Biomed Engin 2014;17:73-93.

45. Thévenaz P, Unser M. Optimization of mutual information for multiresolution image registration. IEEE Trans Image Process 2000;9:2083-99.

46. Pluim JPW, Maintz JBA, Viergever MA. Image registration by maximization of combined mutual information and gradient information. IEEE Trans Med Imaging 2000;19:809-14.

47. Butz T, Thiran JP. Affine Registration with Feature Space Mutual Information. In MICCAI 2001: Medical Image Computing and Computer-Assisted Intervention: MICCAI 2001:549-56.

48. Lee JY, Jeon BS, Paek SH, et al. Reprogramming guided by the fused images of MRI and CT in subthalamic nucleus stimulation in Parkinson disease. Clin Neurol Neurosurg 2010;112:47-53.

49. Barnaure I, Pollak P, Momjian S, et al. Evaluation of electrode position in deep brain stimulation by image fusion (MRI and CT). Neuroradiology 2015;57:903-8.

50. Klein S, Staring M, Murphy K, et al. elastix: A Toolbox for Intensity-Based Medical Image Registration. IEEE Trans Med Imaging 2010;29:196-205.

51. van Eijnatten M, van Dijk R, Dobbe J, et al. CT image segmentation methods for bone used in medical additive manufacturing. Med Eng Phys 2018;51:6-16.

52. Manniesing R, Velthuis BK, van Leeuwen MS, et al. Level set based cerebral vasculature segmentation and diameter quantification in CT angiography. Med Image Anal 2006;10:200-14.

53. Shattuck DW, Leahy RM. Brainsuite: An automated cortical surface identification tool. Med Image Anal 2002;6:129-42.

54. Fischl B, Van Der Kouwe A, Destrieux C, et al. Automatically Parcellating the Human Cerebral Cortex. Cereb Cortex 2004;14:11-22.

55. Yiannakas MC, Mustafa AM, De Leener B, et al. Fully automated segmentation of the cervical cord from T1weighted MRI using PropSeg: Application to multiple sclerosis. NeuroImage Clin 2015;10:71-7.

56. Sun R, Wang K, Guo L, et al. A potential field segmentation based method for tumor segmentation on multi-parametric MRI of glioma cancer patients. BMC Med Imaging 2019;19:48.

57. Pereira S, Pinto A, Alves V, et al. Brain Tumor Segmentation Using Convolutional Neural Networks in MRI Images. IEEE Trans Med Imaging 2016;35:1240-51.

58. Gargiulo P, Árnadóttir I, Gíslason M, et al. New Directions in 3D Medical Modeling: 3D-Printing Anatomy and Functions in Neurosurgical Planning. J Healthc Eng 2017;2017:8.

59. Konakondla S, Brimley CJ, Sublett JM, et al. Multimodality 3D Superposition and Automated Whole Brain Tractography: Comprehensive Printing of the Functional Brain. Cureus 2017;9:e1731.

60. Kikinis R, Pieper SD, Vosburgh KG. 3D Slicer: A Platform for Subject-Specific Image Analysis, Visualization, and Clinical Support. In: Intraoperative Imaging and ImageGuided Therapy. New York, NY: Springer, 2014:277-89.

61. Wang D, Ma D, Wong ML, et al. Recent advances in surgical planning and navigation for tumor biopsy and resection. Quant Imaging Med Surg 2015;5:640-8. 
62. Fischl B, Sereno MI, Dale AM. Cortical surface-based analysis. I. Segmentation and Surface Reconstruction. Neuroimage 1999;9:195-207.

63. Stava O, Vanek J, Benes B, et al. Stress Relief: Improving Structural Strength of 3D Printable Objects. Available online: http://hpcg.purdue.edu/bbenes/papers/ Stava2012sigg.pdf

64. Huang X, Liu Z, Wang X, et al. A small 3D-printing model of macroadenomas for endoscopic endonasal surgery. Pituitary 2019;22:46-53.

65. Goel A, Jankharia B, Shah A, et al. Three-dimensional models: an emerging investigational revolution for craniovertebral junction surgery. J Neurosurg Spine 2016;25:740-4.

66. Sheth R, Balesh ER, Zhang YS, et al. Three-Dimensional Printing: An Enabling Technology for IR. J Vasc Interv Radiol 2016;27:859-65.

67. Xu W-H, Liu J, Li M-L, et al. 3D printing of intracranial artery stenosis based on the source images of magnetic

Cite this article as: Martín-Noguerol T, Paulano-Godino F, Riascos RF, Calabia-del-Campo J, Márquez-Rivas J, Luna A. Hybrid computed tomography and magnetic resonance imaging 3D printed models for neurosurgery planning. Ann Transl Med 2019;7(22):684. doi: 10.21037/atm.2019.10.109 resonance angiograph. Ann Transl Med 2014;2:74.

68. Hadizadeh DR, Marx C, Gieseke J, et al. High temporal and high spatial resolution MR angiography (4D-MRA). Rofo 2014;186:847-59.

69. Ahmed AK, Pennington Z, Molina CA, et al. Multidisciplinary surgical planning for en bloc resection of malignant primary cervical spine tumors involving 3D-printed models and neoadjuvant therapies: report of 2 cases. J Neurosurg Spine 2019. [Epub ahead of print].

70. Bishop N, Boone D, Williams K-L, et al. Development of a Three-dimensional Printed Emergent Burr Hole and Craniotomy Simulator. Cureus 2019;11:e4373.

71. Griffith B, Jain R. Perfusion Imaging in Neuro-Oncology: Basic Techniques and Clinical Applications. Magn Reson Imaging Clin N Am 2016;24:765-79.

72. Kikuchi K, Hiwatashi A, Togao O, et al. Correlation between arterial spin-labeling perfusion and histopathological vascular density of pediatric intracranial tumors. J Neurooncol 2017;135:561-9. 